\title{
Dimethyl formamide as dispersing agent for electrophoretically deposited of multi-walled carbon nanotubes
}

\begin{abstract}
Dispersing agents used during electrode position process are required to form stable suspension of solution at room temperature for controllable thickness of deposited materials. The solvent selected for dispersion of multi-walled carbon Nanotubes (MWCNTs) are mixture of acetone and ethanol, dimethyl formamide (DMF), N-methyl2-pyrrolidone (NMP) and distilled water were investigated. Among these solvents, DMF shows better suspension stability and performed well during electrophoretic deposition (EPD) of MWCNTs on nickel substrate. Analysis by scanning electron microscopy (SEM) shows the maximum of $4.78 \mu \mathrm{m}$ thick of MWCNTs deposited on nickel substrate when operated under150V applied voltage, 10 minutes deposition time for $0.50 \mathrm{mg} / \mathrm{ml}$ concentration.
\end{abstract}

Keywords: carbon nanotubes, dimethyl formamide, electrophoretic deposition
Volume I Issue I - 2016

\author{
Abdul Rahman Mohamed,' Raihana Bahru,' \\ Wei-Ming Yeoh, ${ }^{2}$ Khatijah Aisha Yaacob ${ }^{3}$ \\ 'School of Chemical Engineering, Universiti Sains Malaysia, \\ Malaysia \\ ${ }^{2}$ Department of Petrochemical Engineering, Universiti Tunku \\ Abdul Rahman, Malaysia \\ ${ }^{3}$ School of Materials and Mineral Resources Engineering, \\ Universiti Sains Malaysia, Malaysia
}

Correspondence: Abdul Rahman Mohamed, School of Chemical Engineering, Universiti Sains Malaysia, Malaysia, Tel +60 459964 I0, Fax +60 459410 3, Email chrahman@usm.my

Received: July 28, 2016 | Published: September 16, 2016

\section{Introduction}

Discovery of multi-walled carbon Nanotubes (MWCNTs) since 1991 had extended to the various applications such as thermal interface materials, electrochemical super capacitor and solar cell. ${ }^{1-4}$ Most of the application of MWCNTs relates to the thin film deposition either individually or in composite formation. MWCNTs are very stable and inert substance that required specific condition and environment in order to debundle and stably disperse in solvent media. Besides, the difficulties found when it possess high aspect ratio which causes Nanotubes easily aggregate and the effect of van der Waals attraction between tubes prevent them for suspension. ${ }^{5}$

The methods for MWCNTs dispersion can be chemically or mechanically. Chemical method required fictionalization of MWCNTs using strong acid which introduces the hydrophilicity at their surface, but it also causes structural defects in CNTs. Meanwhile for mechanical method, high shear mixing, Ultrasonication and solvent are used for dispersion. ${ }^{5}$ Cheng $^{6}$ reported the dispersion and debundling of CNTs via sonication process but it resulted in damages to the Nanotubes when operate at high intensity of mixing. With the use of suitable solvent for dispersion, the damage caused by mechanical method can be minimized.

In this study, electrophoretic deposition (EPD) method is used for pristine MWCNTs deposition which operates at room temperature with simple and easy-handling set up. ${ }^{7-9}$ Various solvents were tested for MWCNTs dispersion such as organic solvent (mixture of acetone and ethanol and N-methyl-2-pyrrolidone (NMP) and dimethyl formamide (DMF)), polar solvent (distilled water) and surfactant. ${ }^{10-12}$ The suitable solvent is selected based on solvent type, suspension stability, zeta potential reading and performance for EPD. The aim for this research is to select the most suitable solvent for dispersion and deposition of MWCNTs on nickel substrate using EPD method. The maximum thickness of deposition is high for single layer of deposition.

\section{Experimental procedure}

The MWCNTs were synthesized via catalytic decomposition of methane over bimetallic Co-Mo/MgO catalyst. ${ }^{13}$ They were purified to remove the impurities i.e. catalyst particles and amorphous carbon from as-synthesized MWCNTs before dispersed in the solvents selected which are dimethyl formamide (DMF), N-methyl, 2-pyrrolidone (NMP), mixture of acetone and ethanol, and distilled water. The MWCNTs were dispersed using sonic dismembrator (FISHER SCIENTIFIC) at 50\% amplitude for 10 minutes and final solution concentration was $0.50 \mathrm{mg} / \mathrm{ml}$. The dispersed MWCNTs was kept inside the room temperature undisturbed to examine the stability of the solutions.

The solution was analyzed using UV-Vis spectrometer (SHIMADZU UV-1601) to measure the stability of the solution. The reading of UV-Vis was taken up to the solution settled after fourteen days. After that, the solution which form stable suspension was analyzed using Malvern Instruments Nano Series Zetasizer (model: ZEN 3600) to obtain the zeta potential analysis. The stabilized MWCNTs in suitable solvent was deposited using EPD on nickel substrate, which was cleaned with ethanol before use. The effects on film thickness were studied at $150 \mathrm{~V}$ of applied voltage with 10 minutes of deposition time. The electrode distance were kept constant at $15 \mathrm{~mm}$ apart and connected to power supply (KIKUSUI Regulated DC Power Supply, model PAS500-0.6). The thickness of deposited MWCNTs was analyzed using scanning electron microscopy (SEM; FEI, model Quanta 450 FEG). 


\section{Results and discussion}

Figure 1A shows the dispersion stability of

i. Dimethyl formamide, DMF
ii. N-methyl, 2-pyrrolidone, NMP
iii. Mixture of acetone and ethanol
iv. Distilled water
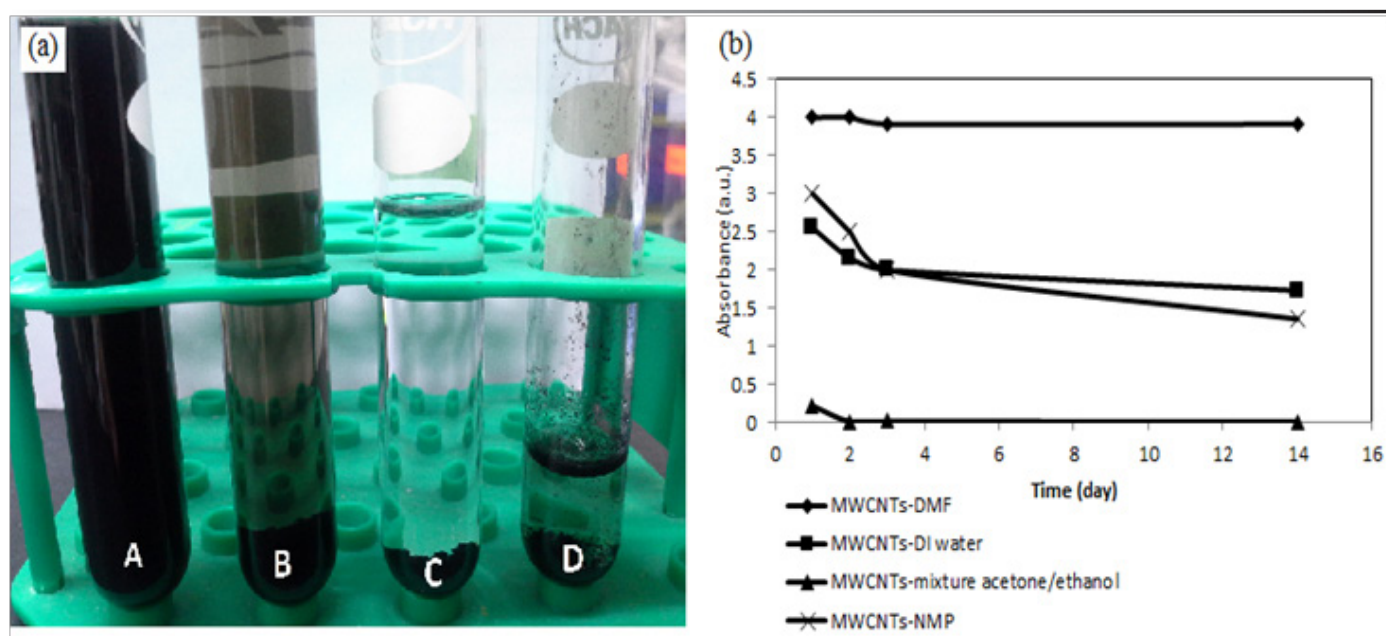

Figure I A Dispersion of MWCNTs in different type of solvents (A) Dimethylformamide, (B) N-methyl, 2-pyrrolidone, (C) mixture of acetone and ethanol, (D) distilled water. B. UV-Vis reading at $500 \mathrm{~nm}$ wavelength.

The purified MWCNTs settled quickly in mixture of acetone and ethanol at 30 minutes after sonication. Meanwhile dispersion of MWCNTs in NMP and distilled water were completely settled after 14 days. Among these solvents, the DMF give the longest suspension stability of MWCNTs which keep suspending up to three months. The suspension stability of MWCNTs is the most important criteria for electrophoretic deposition to pull the particle for electrode position to the substrate, ${ }^{9}$ as high agglomeration tendency of particle in solution inhibit the deposition of particle on substrate.

The suspension stability of MWCNTs is analyzes using UV-Vis at wavelength of $500 \mathrm{~nm}$. Figure $1 \mathrm{~b}$ shows the UV-Vis reading of four types of solvents used in dispersion of MWCNTs with concentration of $0.50 \mathrm{mg} / \mathrm{ml}$. From the graph, the reading of dispersed MWCNTs in DMF is almost constant in 14 days at $3.90-4.00 \mathrm{~A}$. Meanwhile the reading pattern for NMP is decreased from 3.00to 1.36A and similar trend for distilled water, from 2.55 to $1.76 \mathrm{~A}$. The settling time of MWCNTs in mixture of acetone and ethanol is the fastest with the UV-Vis reading at 0.21 to $0.01 \mathrm{~A}$ within 30 minutes after sonication.NMP is mostly applied in dispersing single-walled carbon Nanotubes (SWCNT) as it is reported to enable the control of SWCNT suspension. ${ }^{12,14}$ However, combination of NMP with MWCNTs as seen in the graph, the UV-Vis reading for NMP is initially at 3.00A dropped by $55 \%$ after 14 days. According to the report by Riddick ${ }^{15}$ in Figure 2, when the potential is small, attractive forces may exceed this repulsion and the dispersion may break and flocculate. So, colloids with high zeta potential (negative or positive) are electrically stabilized while colloids with low zeta potentials tend to coagulate or flocculate. Inam ${ }^{16}$ reported that the DMF is good dispersant compare to ethanol for making homogeneous MWCNTs, stable and composite dispersion. Besides, the benefit of using organic compound as dispersant is due to the low boiling point which can remove easily and avoid any residue leave in the deposited film. ${ }^{7}$

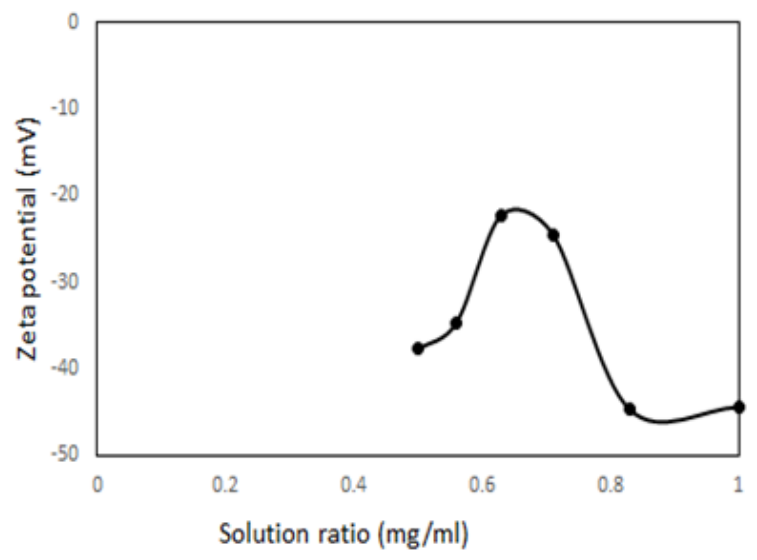

\begin{tabular}{ll}
\hline Stability Characteristics & $\begin{array}{l}\text { Average Zeta } \\
\text { Potential in } \mathrm{mV}\end{array}$ \\
\hline Maximum agglomeration and precipitation & 0 to +3 \\
Range of strong agglomeration and precipitation & +5 to -5 \\
Treshold of agglomeration & -10 to -15 \\
Treshold of delicate dispersion & -16 to -30 \\
Moderate stability & -31 to -40 \\
Fairly good stability & -41 to -60 \\
Very good stability & -61 to -80 \\
Extremely good stability & -81 to -100 \\
\hline
\end{tabular}

Figure 2 Zeta potential profile when increasing the ratio of mass MWCNTs (mg) to volume of dimethylformamide (ml) and table of stability of suspension with relation to zeta potential (on the right). ${ }^{15}$ 
The effect of DMF solution for MWCNTs dispersion was measured using zetasizer and showed in Figure 2, varied in volume 50 to $100 \mathrm{ml}$ gave the ratio with mass of MWCNTs from 0.50 to $1.00 \mathrm{mg} / \mathrm{ml}$. The reading of zeta potential is $-37.73,-34.6,-22.17,-24.47$ and $-44.4 \mathrm{mV}$ for ratio $0.50,0.56,0.63,0.71,0.83$ and $1.00 \mathrm{mg} / \mathrm{ml}$, respectively. The zeta potential value is not constant due to the charge on MWCNTs in DMF solution and it shows that the result obtained is depends on concentration of the solution, same as reported by Tantra. ${ }^{17}$ Based on table by Riddick, ${ }^{15}$ the charge of the particle is more than absolute $30 \mathrm{mV}$ is generally represent sufficient mutual repulsion to result in stability. The ratio of the solution for $0.50,0.56,0.83$ and $1.00 \mathrm{mg} /$ $\mathrm{ml}$ have good stability without tends to form agglomeration while the ratio 0.63 and 0.71 is in the threshold region of either coagulation or dispersion exists.

From the zeta potential analysis, the solutions of MWCNTs were deposited on nickel substrate using electrophoretic deposition method. DMF have high dielectric constant is sufficient to perform during EPD as good conductor besides keep the suspension stable. Figure 3 shows the SEM image of assembled MWCNTs for solution ratio of $0.50,0.56,0.63,0.71$ and $0.83 \mathrm{mg} / \mathrm{ml}$ at $150 \mathrm{~V}$ of applied voltage for 10 minutes of deposition. The table in Figure 3 indicated the value of EDX for spectrum 1 and 2, which represent MWCNTS dominant layer and $\mathrm{Ni}$ dominant layer, respectively. The maximum thickness of deposited MWCNTs for different solution ratio is $4.78 \mu \mathrm{m}$.

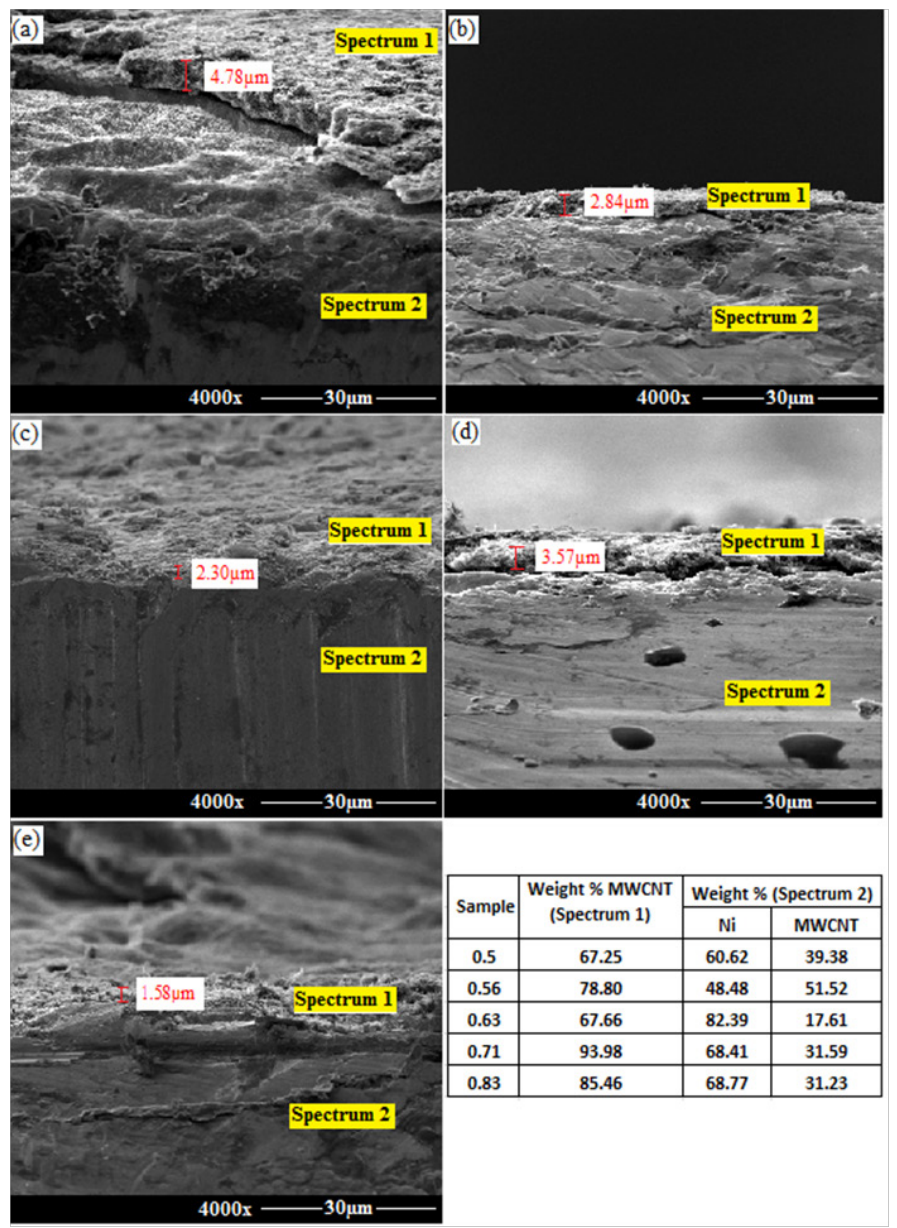

Figure 3 SEM and EDX value for MWCNTs deposited on nickel plate (A) 0.50,(B) 0.56, (C) 0.63, (D) 0.7I, (E) $0.83 \mathrm{mg}$ MWCNTs /ml DMF.

\section{Conclusion}

This investigation confirms that the dimethyl formamide is suitable as dispersing agent for electrophoretically deposited MWCNTs compare to other solvents. The stability result of the DMF-MWCNTs solution exhibit excellent performance for EPD method, which formed stable solution for more than fourteen days. The zeta potential analysis proved the deposition ability of the solution for reading absolute $30 \mathrm{mV}$ and above. The SEM result show the maximum thickness deposited on the nickel substrate which is $4.78 \mu \mathrm{m}$.

\section{Acknowledgements}

None.

\section{Conflict of interest}

The authors declare no conflict of interest.

\section{References}

1. Iijima S. Helical microtubules of graphitic carbon. Nature.1991;354:56 -58 .

2. Fabris $\mathrm{D}$, Rosshirt $\mathrm{M}$, Cardenas $\mathrm{C}$, et al. Application of carbon nanotubes to thermal interface materials: j electron. Packag. 2011;133(2):020902020908 .

3. Song L, Guan Y, Du P, et al. Enhanced efficiency in flexible dyesensitized solar cells by a novel bilayer photoanode made of carbon nanotubes incorporated $\mathrm{TiO}_{2}$ nanorods and branched $\mathrm{TiO}_{2}$ nanotubes. $J$ Sol Energy Mater Sol. 2016;147:134-143.

4. Kim BW, Chung H, Min BK, et al. Electrochemical capacitors based on aligned carbon nanotubes directly synthesized on tantalum substrates. Bull Korean Chem Soc. 2010;31(12):36973702.

5. Nayak S, Bhattacharjee S, Singh BP. A systematic study on the effect of acidic, basic and neutral additives on dispersion of multiwalled carbon nanotubes using a dimethyl formamide solution. Adv Nat Sci Nanosci. and Nanotechnol. 2014;5(4):045005 -0450013.

6. Cheng Q. Dispersion of single-walled carbon nanotubes in organic solvents: doctoral thesis dublin. Dublin Inst of Technol. 2010;176.

7. Sarkar P, Nicholson P. Electrophoretic deposition (EPD): mechanisms, kinetics and applications to ceramics. J Am Ceram Soc. 1996;79(8):1987 -2002 .

8. Besra L, Liu M. A review on fundamentals and applications of electrophoretic deposition (EPD). Prog Mater Sci. 2007;52(1):1-61.

9. Boccaccini AR, Cho J, Roether JA, et al. Electrophoretic deposition of carbon nanotubes. Carbon. 2006;44(15):3149-3160.

10. Zhao L, Gao L. Stability of multi-walled carbon nanotubes dispersion with copolymer in ethanol.Colloids Surfaces A: Physicochem Eng Asp. 2003;224(1-3):127134.

11. Fu Q, Jiu JT, Cao CB, et al. Electrodeposition of carbon films from various organic liquids. Surf Coat Technol. 2000;124(2-3):196-200.

12. Forney MW, Poler JC. Significantly enhanced single-walled carbon nanotube dispersion stability in mixed solvent systems. $J$ Phys Chem C. 2011;115(21):10531-10536.

13. Yeoh WM, Lee KT, Mohamed AR, et al. Production of carbon nanotubes from chemical vapor deposition of methane in a continuous rotary reactor system. Chem Eng Commun. 2012;199(5):600-607.

14. Rahman MM, Younes H, Subramanian N, et al. Optimizing the dispersion conditions of SWCNTs in aqueous solution of surfactants and organic solvents. $J$ of Nanomater. 2014;1-11. 
15. Riddick TM. Control of colloid stability through zeta potential. Livingston Publishing. New York; 1968.

16. Inam F, Yan H, Reece MJ, et al. Dimethylformamide: an effective dispersant for making ceramic-carbon nanotube composites. Nanotechnology. 2008;19(19):195-710.
17. Tantra R, Schulze P, Quincey P. Effect of nanoparticle concentration on zeta-potential measurement results and reproducibility. Particuology. 2010;8(3):279-285. 